\title{
Collision Characteristics between Ultracold Cesium nP Rydberg Atoms
}

\author{
Zhigang Feng*, Xin Zhou, Bo Li, Hongying Gao, Zheng Liu \\ Division of Electronics and Information Technology, National Institute of Metrology, Beijing, China \\ Email: ^fengzg@nim.ac.cn
}

How to cite this paper: Feng, Z.G., Zhou, X., Li, B., Gao, H.Y. and Liu, Z. (2018) Collision Characteristics between Ultracold Cesium $n P$ Rydberg Atoms. Journal of Modern Physics, 9, 820-831. https://doi.org/10.4236/jmp.2018.95053

Received: November 25, 2017

Accepted: April 7, 2018

Published: April 10, 2018

Copyright $\odot 2018$ by authors and Scientific Research Publishing Inc. This work is licensed under the Creative Commons Attribution International License (CC BY 4.0).

http://creativecommons.org/licenses/by/4.0/

\begin{abstract}
We investigate the effect of van der Waals interactions and dipole-dipole interactions on collisional loss rate coefficients of Cs Rydberg $n P$ states, and in detail analyze the variation of collisional loss coefficients under the initial Rydberg atomic velocity and van der Waals interactions. We obtain the total collisional loss coefficients for different $n P$ states, and provide the possible ionization mechanism for the results of experimental observation using our analysis model.
\end{abstract}

\section{Keywords}

Collisional Loss, Rydberg Atoms, Interactions

\section{Introduction}

The ultracold Rydberg atoms have been extensively studied due to their strong and controllable long-rang interactions. The interaction-induced dipole blockade effect has been proposed as an ideal candidate for realizing a scalable quantum logic gate applying in quantum information processing [1] [2]. The dipole blockade effects based on ultracold Rydberg atoms have been experimentally studied for van der Waals interaction case by changing density or the principal quantum number of Rydberg state [3] [4], and for the dipole-dipole interaction case by tuning an electric field [5]. The ultracold Rydberg atoms move only a few percent of typical interatomic spacing on the time scale of experimental interest; in other words, the ultracold Rydberg atoms have smaller thermal kinetic energy relative to their average interaction energies, so ultracold Rydberg atoms provide an alternative platform to study interaction induced collision dynamics and evolution process. Some groups experimentally investigated collision and ionization mechanism of a pair of Rydberg atoms initially excited on the attractive po- 
tential for dipole-dipole interaction case [6], and van der Waals interaction case [7], which will give rise to the faster collisions and even avalanche ionization forming ultracold plasmas [8].

Blackbody radiation (BBR) is known to strongly affect the populations of Rydberg atoms by transitions from initial state to nearby dipole allowed states or BBR-induced direct ionization [9]. Amthor et al. showed that the Rydberg atoms' collisional ionization was also observed on longer time scales when atoms are excited to the repulsive van der Waals potential, which is attributed to the $B B R$ redistribution to the effective attractive interaction potential [10]. And the initial thermal motion of the ultracold Rydberg atoms can significantly shorten the collision time [11]. For a pair of Cs $n P$ atoms, penning ionization and the formation of an ultracold plasma mostly occur on attractive potential for states with $n>42$ [12]. So the long-range potential character of Rydberg atoms determines the collisional and ionization dynamic mechanism. However, above works on collision and ionization induced by Rydberg atomic interactions were mainly focused on the spectroscopic measurements based on the pulsed field ionization techniques, which have proven to be a powerful measuring method for Rydberg atoms and utilized widely. Based on the analysis of the decay curve provides a new method for studying Rydberg atoms interactions and collisional ionization process, which is significantly affected by the Rydberg atomic strong interaction and a weak background DC electric field.

In this paper, we in detail analyze the collisional loss mechanisms induced by Rydberg atoms interactions for different Cs $n P$ states using the theoretical model used before. The validity of this theoretical model has been proved in our previous article by comparing the theoretical and experimental values of collisional loss rate coefficient of Cs $63 S$ state [13], but it is different from this paper: First, for Cs $n S$ Rydberg states, the interaction potentials are repulsive, and we only study the effect of repulsive interactions on collisional loss, and consider simultaneously the BBR effect. For the Cs $n P$ states, the interaction potential only for $\mathrm{n}<42$ is repulsive, but for $\mathrm{n}>42$ is attractive potential. In this paper, we can simultaneously study the collisional loss of $n P$ states for attractive and repulsive case, and compare the effect on collisional loss rate under the same condition, especially the role of BBR in this process for two cases; in addition, the different spontaneous radiation rates, BBR transition rate and their relative ratio for $n S$ and $n P$ Rydberg states will also lead to different collisional loss mechanisms. Second, we investigate the important role of initial van der Waals interaction in total collision process for attractive and repulsive case, in detail analyze the effect on the total collisional loss rate, and improve the analytical formula for both cases. This content has not been studied in previous papers.

\section{Theoretical and Analysis}

The collisional characteristics of cold atoms are conventionally determined from the analysis of the decay curve. We can obtain the analytic solution of the time 
evolution of the Rydberg atoms number [13]

$$
N_{R y}(t)=\frac{\left(4 \pi w_{R y}^{2}\right)^{3 / 2} \alpha^{\prime} N_{R y}(0)}{\left[\left(4 \pi w_{R y}^{2}\right)^{3 / 2} \alpha^{\prime}+\beta N_{R y}(0)\right] \mathrm{e}^{\alpha^{\prime} t}-\beta N_{R y}(0)}
$$

where

$$
\alpha^{\prime}=\alpha+\beta_{R y-G} N_{G}\left[\frac{1}{2 \pi\left(w_{R y}^{2}+w_{G}^{2}\right)}\right]^{3 / 2}+\beta_{R y-e} N_{e}\left[\frac{1}{2 \pi\left(w_{R y}^{2}+w_{e}^{2}\right)}\right]^{3 / 2}
$$

Here, $\alpha$ is the loss rate coefficient including the spontaneous radiation rate $\Gamma_{S p o n}$ and blackbody radiation rate $\Gamma_{B B R}$. The blackbody radiation rate include not only the blackbody radiation transition rates to all nearby Rydberg states through absorption and stimulated emission, but also the direct blackbody radiation photoionization rate. $\beta\left(\beta_{R y-G}\right)$ is the loss rate coefficient caused by collisions between ultracold Rydberg-Rydberg (Rydberg-ground) atoms; $\beta_{R y-e}$ is the loss rate coefficient caused by collisions between ultracold Rydberg atoms and electrons. $N$ is the atomic number; subscript $R y(G)$ denotes Rydberg (ground) state; $W_{R p} W_{G}$ and $w_{e}$ are the waist radius of Rydberg atoms, cold atoms sample and ionized electrons, respectively.

The value of dipole elements between Rydberg-ground atoms is two orders of magnitude smaller than that of between Rydberg atoms, and decrease with increasing of $n$, so the collisional loss rate induced by the weak interactions between Rydberg-ground atoms has negligible effect on the total collisional loss rate. Although the large geometrical cross section of Rydberg atoms will lead to the large quenching collisional loss for higher Rydberg states, but has weak contribution to the total collisional loss rate for $n<50$ [13], so the Equation (2) can be rewritten as

$$
\alpha^{\prime}=\alpha+\beta_{R y-e} N_{e}\left[\frac{1}{2 \pi\left(w_{R y}^{2}+w_{e}^{2}\right)}\right]^{3 / 2}
$$

In order to investigate the collisional loss induced by Rydberg atomic interactions, we detailed analyze the relation between collisional loss rate coefficient and interactions. The collisional loss rate coefficient can be written as $\beta=\left\langle\sigma_{R y} v_{R y}\right\rangle$, the $\sigma_{R Y}$ and $v_{R Y}$ are the collisional cross section and relative collisional velocity of ultracold Rydberg atoms, both depend on their van der Waals interaction or dipole-dipole interaction. The collisional cross section induced by interactions given by [14]

$$
\sigma_{i}=\pi\left(\frac{f(n) C_{n}}{m_{C s} v_{e s c} v_{c}}\right)^{2 / n}
$$

where $m_{C s}$ is Cs atom mass; $v_{c} \approx\left(8 k_{B} T / \pi m_{C s}\right)^{1 / 2}\left(k_{B}\right.$ and $T$ are the Boltzmann constant and the temperature of cold atoms) is the average velocity of cold atoms; $C_{n}$ is the interaction coefficient, which is given by theoretical calculations [15]; $f(3)=4$ and $f(6)=15 \pi / 8$ are $f(n)$ values for van der Waals interaction and 
dipole-dipole interaction case. For a pair of Rydberg atoms separated by a distance $R$, they will experience a force and be accelerated toward (away from) each other if they are initially excited on attractive (repulsive) potentials. The force is the gradient of the interaction potential, the relative accelerations caused by van der Waals interaction force and dipole-dipole interaction force can be written as $a_{v d W} \approx 6 C_{6} /\left(m_{C S} R^{7}\right)$ and $a_{D D} \approx 3 C_{3} /\left(m_{C S} R^{4}\right)$, respectively. $v_{e s c}$ is a minimum escape velocity of Rydberg atomic from center to edge of average interaction region under corresponding acceleration, i.e., $V_{\text {esc }}=(2 a r)^{1 / 2}$, the average interaction region is approximately equal to the Rydberg excitation volume with the average radius $r$, in actual experiment, the overlap between trapping laser beam and the Rydberg excitation laser beam defines an effective Rydberg excitation volume. We also obtain the relative collisional velocity between Rydberg atoms under corresponding acceleration, $V_{R y}=(2 a R)^{1 / 2}$, the detailed analysis process has been described in our previous work [14]. Combining the above relations, the corresponding analytic solution of the collisional loss rate coefficient $\beta_{v d W}$ and $\beta_{D D}$ induced by van der Waals interaction and dipole-dipole interaction can be written as

$$
\begin{gathered}
\beta_{v d W}=\sqrt{2} \pi\left(\frac{45 \pi}{4 m_{C s}^{2} \nu_{c}(2 r)^{1 / 2} R^{5 / 2}}\right)^{1 / 3} C_{6}^{2 / 3} \\
\beta_{D D}=\sqrt{2} \pi\left(\frac{48}{2 r v_{c}^{2} m_{C s}^{3} R}\right)^{1 / 6} C_{3}^{5 / 6}
\end{gathered}
$$

Due to the existence of BBR, some Rydberg atoms in initial $n P$ state will be gradually transferred to nearby dipole-allowed $n^{\prime} D$ and $n S$ states, so the initial weak van der Waals interaction between Rydberg atoms also gradually evolve into strong dipole-dipole interaction between initial $n P$ state and nearby dipole-allowed states, these strong attractive dipole-dipole interactions will give rise to faster collision and even ionization between Rydberg atoms. In order to obtain the effective collisional loss rate coefficient $\beta_{D D}^{\text {eff }}$ including the contributions of all larger dipole-allowed states, we use an effective coefficient $C_{3}^{\text {eff }}=\sum p_{i} \mu_{i}^{2}$ instead of $C_{3}$ in Equation (6), where $\mu_{i}$ is dipole matrix elements between initial $n P$ state and nearby dipole-allowed states, $p_{i}$ is the transition probability from the initial $n P$ Rydberg atoms to these dipole-allowed states, which is proportional to the strength of transition dipole moments. The effective collisional loss rate coefficient $\beta_{D D}$ and $\beta_{v d W}$ of $n P_{3 / 2}$ at $3 \mu \mathrm{m}$ atoms separation as a function of $n$ are shown in Figure 1. The corresponding parameters for these and following calculations including $r=220 \mu \mathrm{m}$ and $T=100 \mu \mathrm{K}$. The collisional loss rate coefficients induced by the interactions between $n P_{3 / 2}$ and some nearby larger dipole-allowed states for $n P_{3 / 2}-n S_{1 / 2}, n P_{3 / 2}-(n+1) S_{1 / 2}$ and $n P_{3 / 2}-(n-$ 1) $D_{5 / 2}$ are also shown in Figure 1. It clear seen that the effective collisional loss rate coefficient induced by dipole-dipole interactions are much larger than that of the van der Waals interactions at the same state, which indicate that the strong dipole-dipole interactions between initial $n P$ and dipole-allowed states 


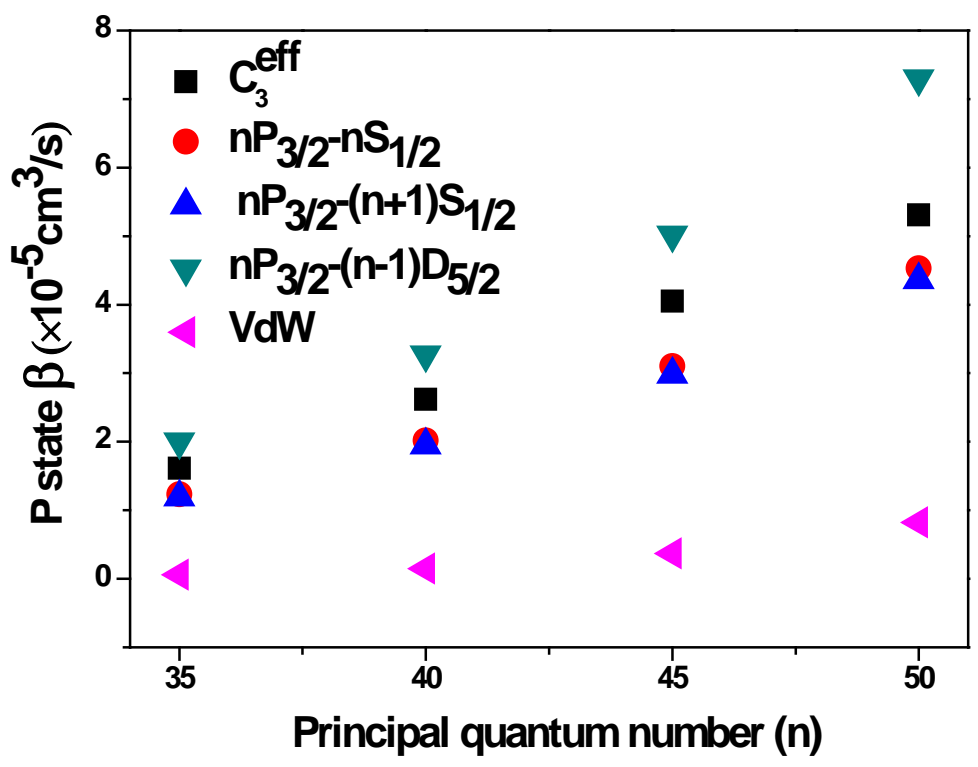

Figure 1. The variation of $\beta_{v d W}$ the effective $\beta_{D D}$ of $n P_{3 / 2}$ and with some nearby larger dipole-allowed states as a function of $n$ at $R=3 \mu \mathrm{m}$.

are the dominant collisional loss mechanism. Because the values of dipole matrix element between initial $n P$ and nearby dipole-allowed states increase with increasing of $n$, and BBR transitions rates gradually equal to even exceed the corresponding spontaneous decay rates with $n$ increasing, which lead to the effective collisional loss rate coefficient slightly nonlinear increase.

In Figure 1, we just separately analyzed the variation of the collisional loss rate coefficient with $n$ for van der Waals interactions and dipole-dipole interactions case, we did not investigate the influence of atomic separation variation induced by the initial Rydberg atomic interactions on collisional loss rate coefficient, and we also require obtain the total collisional loss rates coefficient. For the Cs $n P$ state, the interaction potentials are repulsive potential for $\mathrm{n}<42$, while $\mathrm{n}>42$ for the potential are attractive [12]. For $\mathrm{n}<42$ sates, many experiments observe the appearance of ionized ions on typical $10 \mu$ selay time $t$ [10] [12] if ultracold Rydberg atoms initially excited on the repulsive van der Waals potential, in other words, this delay time is the sum of Rydberg atomic transition time form initial repulsive $n P$ state to dipole-allowed states, and their collision time under attractive dipole-dipole interactions between $n P$ state and dipole-allowed states. The interatomic separation will increase under their repulsive van der Waals forces, which maybe affect the latter interaction strength and collisional distance between $n P$ state and dipole-allowed states. For $n>42$ states, the interatomic separation will decrease under their initial attractive van der Waals forces, and even sharp decrease because the initial weak van der Waals interactions forces gradually evolve into the strong dipole-dipole interactions forces, the average collision time under their initial attractive van der Waals forces equal to, even shorter than the time of the BBR redistribution, which maybe lead to the contributions of interactions between $n P$ state and dipole-allowed states 
gradually diminished, so we must investigate the effect of atoms separation variation on collisional loss rate under initial van der Waals interactions.

In order to obtain the moving distance of atoms compare to the initial atoms separation, we need to consider the relative acceleration of Rydberg atoms pair under initial interaction force and initial velocity of ultracold Rydberg atoms, which is given by $\Delta R=v_{c} t+1 / 2 a t^{2}$. Figure 2 shows the variation of $\Delta R$ with time for different Rydberg states with $3 \mu \mathrm{m}$ initial atomic separation, the initial Rydberg atomic velocity approximately equal to average velocity of cold atoms $v_{\mathcal{C}}$ which is about $18 \mathrm{~cm} / \mathrm{s}$ at cold atoms temperature of $100 \mu \mathrm{K}$. It clear seen that the $\Delta R$ rapidly increase with delay time and even much greater than their initial atoms separation for higher $n$. For lower Rydberg states, the weak interactions give rise to relatively small distance even if long delay time. But the initial velocity of ultracold Rydberg atoms plays an important role, which will give rise to the relative moving distance up to about $1 \mu \mathrm{m}$ at typical $5 \mu$ s delay time, the similar behaviors are experimental investigated for Rb Rydberg atoms [11]. When we only consider the Rydberg atomic initial velocity effect at $5 \mu$ s delay time, the variation of the $\beta_{v d W}$ with $n$ are shown in Figure 3, the value of $C_{6}$ is the average value of all molecular states. The Rydberg atomic separation after $5 \mu$ s delay time will gradually increase to about $4 \mu \mathrm{m}$ for $n<42$ and gradually decrease to about $2 \mu \mathrm{m}$ for $n>42$ because their different interaction characters. In contrast to the results of the fixed initial atoms separation $R=3 \mu \mathrm{m}$, we can see that the collisional loss coefficient $\beta_{v d W}$ increases with the decreasing of $R$ for $n>42$, and have slight decrease for $n<42$ after the same change of $R$, which are shown in Figure 3 with green triangles and red circles, respectively. For $n>42$, the interatomic separation will rapidly decrease due to their attractive van der Waals interactions, which also lead to larger collisional loss rate coefficient $\beta_{D D}$ compare to the initial atoms separation if the BBR has been occurred. Analogous to the

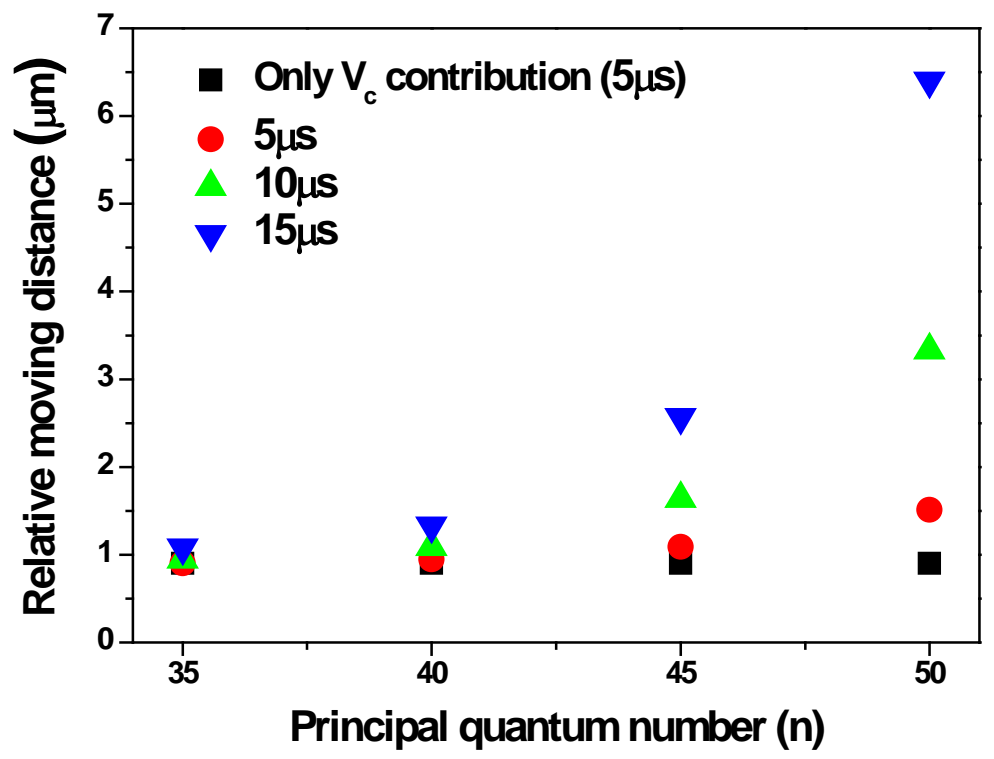

Figure 2. The variation of $\Delta R$ with delay time for different Rydberg states. 


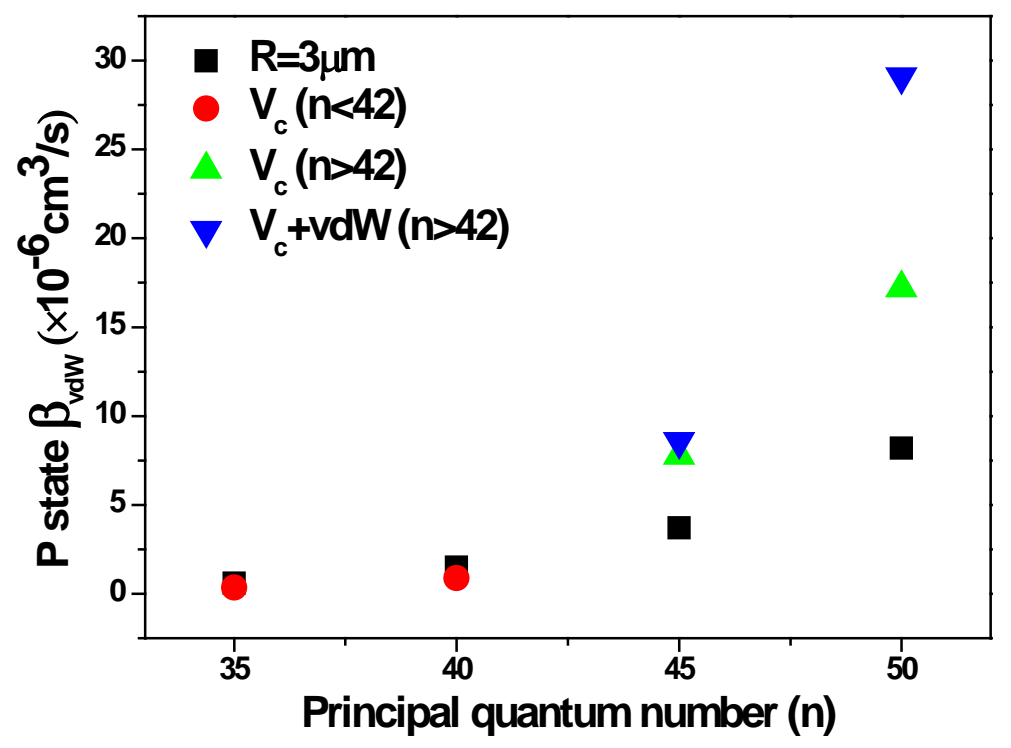

Figure 3. $\beta_{v d W}$ as a function of $n$ for different effects contributions, compared to fixed initial atomic separation of $3 \mu \mathrm{m}$ for all states. The green triangles and red circles only include the contribution of initial Rydberg atomic velocity; the blue triangles consider the contributions of initial Rydberg atomic velocity and van der Waals interactions.

above results and analysis, the variation of the $\beta_{D D}$ with $n$ due to the change of interatomic separation induced by initial velocity are also shown in Figure 4, which have similar results with the van der Waals interactions case.

When we simultaneously consider the initial Rydberg atomic velocity and the van der Waals interactions at the same delay time, because the relative acceleration between ultracold Rydberg atoms will rapidly increase with $n$ scaling as $n^{11}$, the relative moving distances of ultracold Rydberg atoms for high states are much more than that of the low states at the same delay time, which will lead to more obviously changes of interactions with $n$. The variation of the $\beta_{v d W}$ considering the above two effects for $n>42$ are shown in Figure 3 with blue triangles. We can see that the collisional loss rate coefficient obviously increase due to faster decrease of the interatomic separation under their initial attractive van der Waals interactions, compare to the case of only considering the initial Rydberg atomic velocity contribution. The similar variation of the $\beta_{D D}$ for $n>42$ under the effect of the above two factors are also shown in Figure 4 with red circles. Due to slightly increase of atomic separation induced by the weak repulsive van der Waals interactions $n<42$ at this short and even longer delay time, which will give rise to the negligible variation of the collisional loss rate coefficient, so we does not give these corresponding results in Figure 3 and Figure 4.

The total collisional loss rates induced by ultracold Rydberg-Rydberg interactions not only include the attractive (repulsive) interactions between initial $n P$ states, but also the attractive dipole-dipole interactions between $n P$ and nearby dipole-allowed states. Whether Rydberg atomic pairs initially excited on repulsive or attractive potential, the BBR redistribution effect always exists and must 


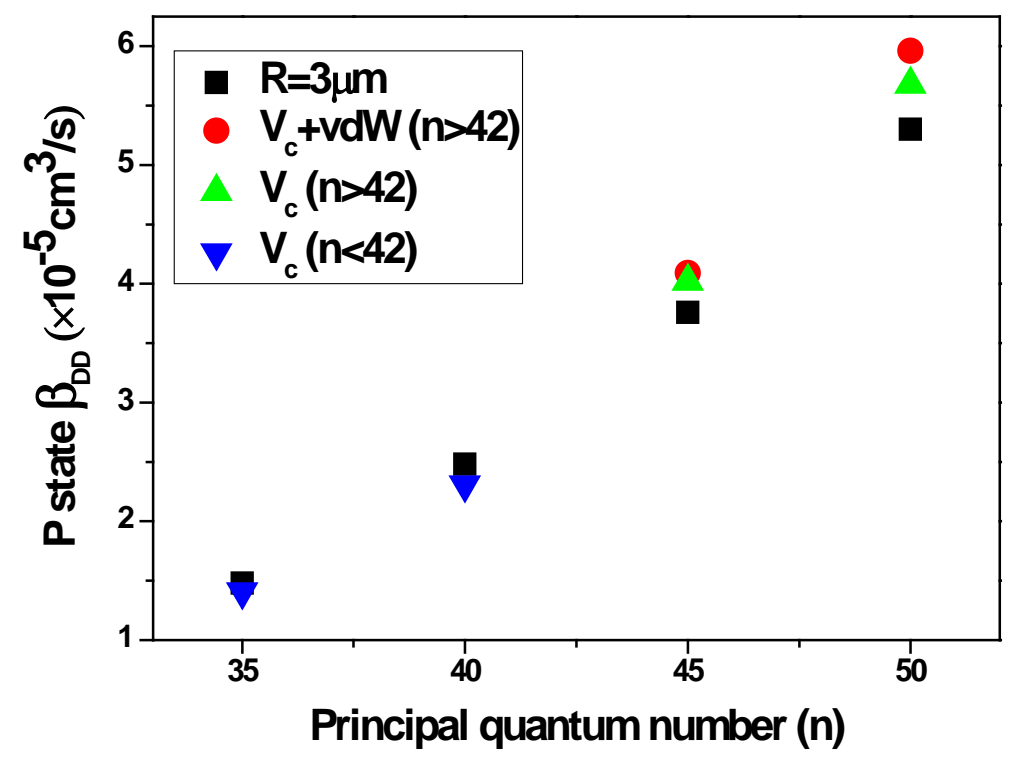

Figure 4. $\beta_{D D}$ as a function of $n$ for different effects contributions, compared to fixed initial atomic separation of $3 \mu \mathrm{m}$ for all states. The triangles only include the contribution of initial Rydberg atomic velocity; the red circles consider the contributions of initial Rydberg atomic velocity and van der Waals interactions.

be taken into account, especially for the repulsive potential case. In order to comprehensive consider all Rydberg interactions contributions to collisional loss rate, we define a ratio such that $F=\Gamma_{B B R} / \alpha$, which is the transition ratio from initial $n P$ state to nearby dipole-allowed states induced by BBR, compare to the total decay rates, the corresponding values of $\Gamma_{B B R}$ and $\alpha$ are obtained by theoretical calculations from reference [9]. For $n>42$ attractive potential case, Rydberg atoms will accelerate toward each other, collide and even ionize under the initial attractive van der Waals interactions, the average collision time at $R=3 \mu \mathrm{m}$ is about $8 \mu$ s for $n=45$ by $\tau_{v d W} \approx 0.2156 R_{0}^{4}\left(C_{6} / m_{C S}\right)^{-1 / 2}$ [7], which is much shorter than the corresponding spontaneous emission lifetime and the effective lifetime at room temperature of $300 \mathrm{~K}$ [16]. This relative long collisional time will lead to most atoms been involved in the collisional process under initial attractive van der Waals interactions before BBR occurrence, and then gradually experiences the attractive dipole-dipole interactions between $n P$ and nearby dipole-allowed states. So the total collisional loss rate coefficient of ensemble atoms system for $\mathrm{n}>42$ can be written as

$$
\beta_{T}=\eta_{M}\left(\beta_{v d W}+F \beta_{D D}^{e f f}\right)
$$

where $\eta_{M}$ is enhancement factor of many-body effects because Rydberg atoms in many atoms system maybe collide each other several times [17], we consider many-body effects with $\eta_{M}=10$ in the following calculations; The van der Waals interactions will introduce gradually increasing contribution for the total collisional loss rate coefficient, so the attractive van der Waals interactions between initial $n P$ states for $n>42$ have non-negligible effect on the total collisional loss 
compared to the strong attractive dipole-dipole interactions between initial $n P$ and nearby dipole-allowed states.

For $n<42$ repulsive potential case, Rydberg atoms will move away from each other under the initial repulsive van der Waals interactions, the collisional ionization does not occur. The collisional probability of ultracold Rydberg atoms initially excited on attractive potential is two orders of magnitude higher than that of repulsive potential case even consider the BBR redistribution effect [18], so we only need to consider the attractive dipole-dipole interactions between initial $n P$ state and nearby dipole-allowed states for $\mathrm{n}<42$, the corresponding total collisional loss rate coefficient can be written as

$$
\beta_{T}=\eta_{M} F \beta_{D D}^{\text {eff }}
$$

At $3 \mu \mathrm{m}$ initial atoms separation and typical $5 \mu$ selay time, the total collisional loss rate coefficient $\beta_{T}$ of $n P_{3 / 2}$ versus $n$ is shown in Figure 5 with blank blocks. It is clear that the total collisional loss rate obviously enhance with $n$ because of collision effect induced by increasing attractive interactions for $n>42$ compare to $n<42$ case. The total collisional loss rates for $n>42$ include not only the contribution of attractive interactions between initial $n P$ state, but also the contribution of attractive interactions between $n P$ and nearby dipole-allowed states. While the increasing slope of curves for $n>42$ states mainly attributed to the important role of the initial van der Waals interaction, the results for $n>42$ states without considering the atomic separation effect induced by the initial van der Waals interaction are also shown in Figure 5 with red circles. It indicates that ultracold Rydberg atoms for $n>42$ states gradually experience increasing attractive van der Waals interactions, and even stronger dipole-dipole interaction between $n P$ states with decreasing of atomic separation, and simultaneously

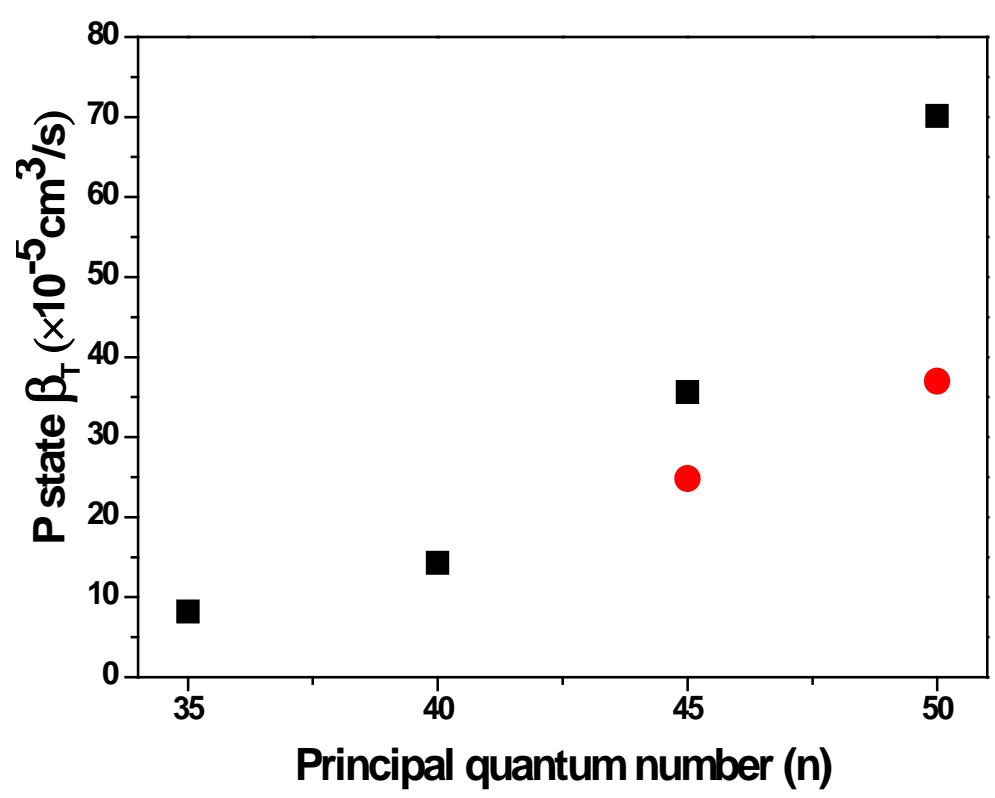

Figure 5. The interaction induced total collisional loss rate coefficient $\beta_{T}$ of $n P_{3 / 2}$ with and without BBR. 
enhance the interactions between initial $n P$ and nearby dipole-allowed states at the longer delay time that the BBR occurred. Because the BBR has occurred before collision induced by attractive van der Waals interactions and stronger dipole-dipole interaction between $n P$ states, the above analysis is suitable to larger Rydberg atomic separation or the lower Rydberg density case. For high Rydberg density, the smaller initial Rydberg atomic separation will lead to strong attractive van der Waals interactions between initial $n P$ state and the rapid decrease of Rydberg atomic separation, then the attractive van der Waals interactions will quickly evolve into stronger dipole-dipole interactions before BBR transition occurrence, we do not consider the contribution of interactions between initial $n P$ and nearby dipole-allowed states, because the Rydberg atoms have been collision and ionization under initial attractive van der Waals interactions and latter stronger dipole-dipole interactions between initial $n P$ state at shorter delay time than that of BBR transition occurrence.

The attractive interaction case have higher ionization probabilities than that the repulsive case due to their different collisional mechanisms, in actual experiment, the collision ionization characteristics have significantly difference for $n>42$ and $n<42$ at longer delay time [12]. As an example, we estimate the Cs $40 P$ collisional ionization rate using our above model, and compare with the measured results from the reference [12]. For experimental results of $40 P$, the density of produced ions varies linearly with initial Rydberg atoms density, the ratio is about $2 \%$. First, the ionization maybe come from BBR photoionization and collisions between the captured electrons and Rydberg atoms [19], the calculated corresponding ionization rates of $40 P$ are about $660 \mathrm{~s}^{-1}$ [20] and $600 \mathrm{~s}^{-1}$ [13] respectively, which are far smaller than the experimental observed value of $2400 \mathrm{~s}^{-1}$. Second, we consider the interaction induced the collisional ionization rate, the initial atomic separation value $R$ is about $3 \mu \mathrm{m}$ for the Rydberg density $\rho=2.5 \times 10^{9} \mathrm{~cm}^{-3}$ by $R=\left(\frac{1}{(2 \pi)^{3 / 2} \rho}\right)^{1 / 3}$, which will give rise to about $10 \%$ of the Rydberg atoms close together [12], the theoretical value of the total collisional loss rate coefficient for $40 P$ is about $1.4 \times 10^{-5} \mathrm{~cm}^{3} / \mathrm{s}$ using our model, and the corresponding collisional loss rate is about $3.5 \times 10^{4} \mathrm{~s}^{-1}$. We can extract the ionization rate is about $700 \mathrm{~s}^{-1}$ with ionization ratio of $2 \%$, even for higher Rydberg density of $4.0 \times 10^{9} \mathrm{~cm}^{-3}$, the ionization rate is only about $1120 \mathrm{~s}^{-1}$, which is also smaller than the experimental value. For $n<42$, we only consider the contribution of the initial Rydberg atomic velocity to the atomic separation, and think that the weak interactions between Rydberg atoms make atoms separation almost unchanged before BBR transition occurrence. When some close atoms pairs exist at the beginning, they will repeatedly approach each other on long delay time, but the atoms redistributed to nearby dipole-allowed states by BBR will have the chance to collide with these repelled and coming closer atoms, the smaller interatomic distance will give rise to faster collisions due to their stronger dipole-dipole interactions [10], which could contribute to an increased colli- 
sional loss rate, so the van der Waals interaction play an important role in collision process for repulsive case. If we consider the effect of initial van der Waals interaction and the initial atoms velocity on interatomic distance, for initial Rydberg density of $4.0 \times 10^{9} \mathrm{~cm}^{3} / \mathrm{s}$, the distance between $40 P$ Rydberg atoms under initial interatomic interaction will increase $2 \mu \mathrm{m}$ on $10 \mu$ s delay time, and a fraction of these repelled atoms may closer to redistributed atoms in nearby dipole-allowed states, compared to their initial atomic separation, which will lead to the faster collisions and ionizations under their stronger dipole-dipole interactions, the calculated ionization rate is about $2240 \mathrm{~s}^{-1}$ with ionization ratio of $2 \%$. In additional, the stray fields from ions can transfer more initial Rydberg atoms to nearby attractive dipole-coupled Rydberg state, and tune their interaction strengths by potential curve determining atomic collisional properties; the present of electric field also can accelerate the impact ionization of Rydberg-electron.

\section{Conclusion}

We in detail analyze the different collisional loss mechanisms of Cs $n P$ Rydberg atoms, present simple analytical formula to estimate these collisional loss rate coefficients for the van der Waals and dipole-dipole case, respectively, estimate Cs $40 P$ collisional ionization rate using our above model, and compare it with the previous measured results, which have the important guiding significance for analyzing the experimental results.

\section{Acknowledgements}

This work was supported by the Fundamental Research Fund Project of NIM (Grant No. 27-AKY1705) and the Exploration and Innovation Project of NIM (Grant No. 27-AKYCX1604).

\section{References}

[1] Jaksch, D., Cirac, J.I., Zoller, P., Rolston, S.L., Côté, R. and Lukin, M.D. (2000) Physical Review Letters, 85, 2208. https://doi.org/10.1103/PhysRevLett.85.2208

[2] Lukin, M.D., Fleischhauer, M., Cote, R., Duan, L.M., Jaksch, D., Cirac, J.I. and Zoller, P. (2001) Physical Review Letters, 87, Article ID: 037901. https://doi.org/10.1103/PhysRevLett.87.037901

[3] Singer, K., Reetz-Lamour, M., Amthor, T., Marcassa, L.G. and Weidemüller, M. (2004) Physical Review Letters, 93, Article ID: 163001. https://doi.org/10.1103/PhysRevLett.93.163001

[4] Tong, D., Farooqi, S.M., Stanojevic, J., Krishnan, S., Zhang, Y.P., Côté, R., Eyler, E.E. and Gould, P.L. (2004) Physical Review Letters, 93, Article ID: 063001.

[5] Vogt, T., Viteau, M., Zhao, J.M., Chotia, A., Comparat, D. and Pillet, P. (2006) Physical Review Letters, 97, Article ID: 083003.

Vogt, T., Viteau, M., Chotia, A., Zhao, J.M., Comparat, D. and Pillet, P. (2007) Physical Review Letters, 99, Article ID: 073002.

[6] Li, W., Tanner, P.J. and Gallagher, T.F. (2005) Physical Review Letters, 94, Article ID: 173001 . 
[7] Amthor, T., Reetz-Lamour, M., Westermann, S., Denskat, J. and Weidemüller, M. (2007) Physical Review Letters, 98, Article ID: 023004. https://doi.org/10.1103/PhysRevLett.98.023004

[8] Robert-de-Saint-Vincent, M., Hofmann, C.S., Schempp, H., Günter, G., Whitlock, S. and Weidemüller, M. (2013) Physical Review Letters, 110, Article ID: 045004. https://doi.org/10.1103/PhysRevLett.110.045004

[9] Beterov, I.I., Tretyakov, D.B. and Ryabtsev, I.I. (2007) Physical Review A, 85, Article ID: 052720 .

[10] Amthor, T., Reetz-Lamour, M., Giese, C. and Weidemüller, M. (2007) Physical Review A, 76, Article ID: 054702. https://doi.org/10.1103/PhysRevA.76.054702

[11] Park, H., Shuman, E.S. and Gallagher, T.F. (2011) Physical Review A, 84, Article ID: 052708. https://doi.org/10.1103/PhysRevA.84.052708

[12] Viteau, M., Chotia, A., Comparat, D., Tate, D.A., Gallagher, T.F. and Pillet, P. (2008) Physical Review A, 78, Article ID: 040704. https://doi.org/10.1103/PhysRevA.78.040704

[13] Feng, Z.G., Zhao, K.J., Miao, J.Y., Li, D.F., Yang, Z.J., Wu, Z.C., He, Z., Zhao, J.M. and Jia, S.T. (2016) Laser Physics, 26, Article ID: 095502.

Feng, Z.G., Zhang, H., Che, J.L., Zhang, L.J., Zhao, J.M., Li, C.Y. and Jia, S.T. (2011) Physical Review A, 83, Article ID: 042711. https://doi.org/10.1103/PhysRevA.83.042711

[14] Steane, A.M., Chowdhury, M. and Foot, C.J. (1992) Journal of the Optical Society of America B, 9, 2142-2158. https://doi.org/10.1364/JOSAB.9.002142

[15] Singer, K., Stanojevic, J., Weidemüller, M. and Côté, R. (2005) Journal of Physics B: Atomic, Molecular and Optical Physics, 38, S295. https://doi.org/10.1088/0953-4075/38/2/021

[16] Beterov, I.I., Ryabtsev, I.I., Tretyakov, D.B. and Entin, V.M. (2009) Physical Review $A, 79$, Article ID: 052504. https://doi.org/10.1103/PhysRevA.79.052504

[17] Mourachko, I., Li, W. and Gallagher, T.F. (2004) Physical Review A, 70, Article ID: 031401(R). https://doi.org/10.1103/PhysRevA.70.031401

[18] Amthor, T., Denskat, J., Giese, C., Bezuglov, N.N., Ekers, A., Cederbaum, L.S. and. Weidemüller, M. (2009) The European Physical Journal D, 53, 329-335. https://doi.org/10.1140/epjd/e2009-00119-4

[19] Robinson, M.P., Laburthe Tolra, B., Noel, M.W., Gallagher, T.F. and Pillet, P. (2000) Physical Review Letters, 85, 4466-4469. https://doi.org/10.1103/PhysRevLett.85.4466

[20] Beterov, I.I., Ryabtsev, I.I., Tretyakov, D.B., Bezuglo, N.N. and Ékers, A. (2008) Journal of Exprimental and Theoretical Physics, 107, 20-27. https://doi.org/10.1134/S1063776108070029 